DOE Final Report August, 2004

Thomas Killian, DOE grant \# DE-F6-03-01ER54659

My research group at Rice University uses a combination of atomic and plasma physics techniques to create neutral plasmas that are orders of magnitude colder than have ever been studied before. Through this work, we probe the basic plasma physics of this exotic regime. During the past year, the major components of the experiment were completed. We demonstrated a powerful new diagnostic, optical imaging of the plasma, which led to a paper that was published in Physical Review Letters. (Figure A, Phys. Rev. Lett. 92, 143001 (2004)) This was the central feature of my DOE Junior Faculty Award proposal. DOE funding has been used to support one postdoctoral researcher, multiple graduate students, the principle investigator, apparatus construction, and normal laboratory expenses.
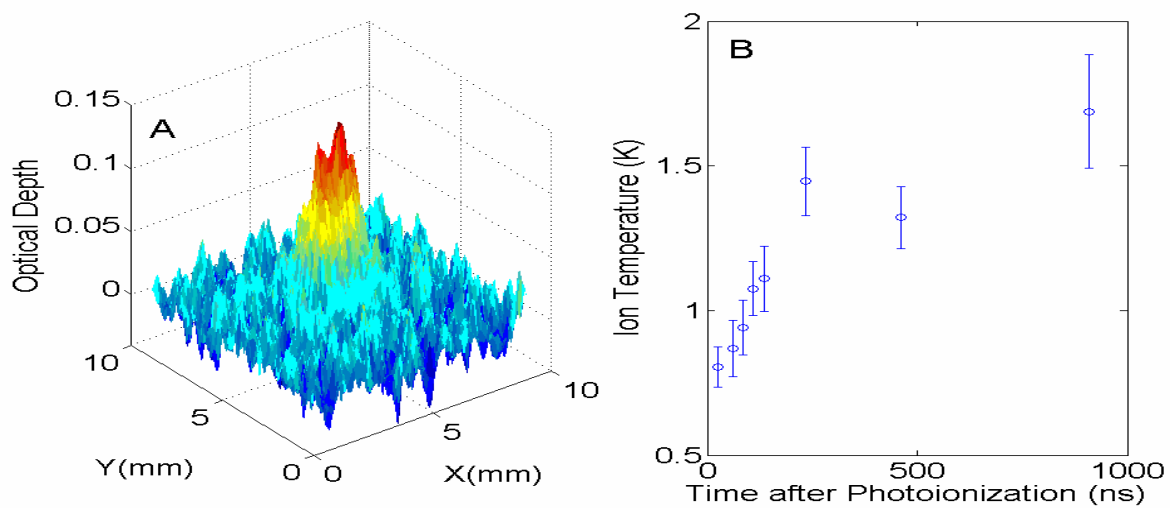

Figure A. Absorption image of an ultracold neutral plasma. Figure B. Ion temperature measured with optical imaging.

We recently submitted an article to Journal of Physics, B, which provides the details of the imaging diagnostic. We are also nearing completion of another manuscript for submission to Physical Review Letters. One of the most striking results in this work is the observation of oscillations in the kinetic energy of the plasma as it equilibrates. (Figure B) This phenomenon has been predicted based on computer simulations of clusters and solid targets irradiated by high-energy lasers, but it has never been seen experimentally. It is a fundamental process in equilibrating strongly-coupled plasmas.

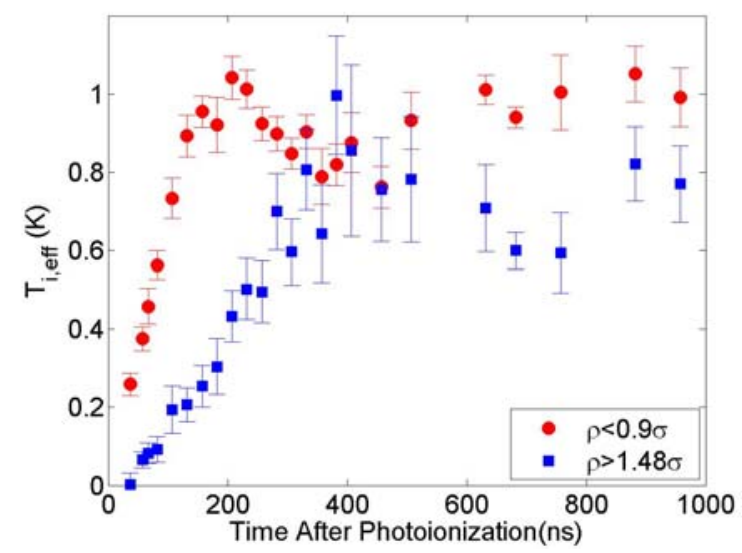

Figure B. Analysis of different regions of the plasma revealed oscillations of the kinetic energy and a lack of global thermal equilibrium in the plasma. The blue circles correspond to an outer region of the plasma. Red circles correspond to the central region of the plasma, which is denser and thus evolves more quickly and becomes hotter. 
This work has already had significant impact. I have been invited to give talks at several international meetings, as well as various seminars and conferences in the United States. I list the invited talks below.

\footnotetext{
"Ultracold Plasmas," $12^{\text {th }}$ International Congress on Plasma Physics, Nice, France, (10/25/04).

"Imaging Ultracold Plasmas," 129th National Meeting of the American Association of Physics
} Teachers, Sacramento, CA, (8/4/04).

"Optically Imaging an Ultracold Strontium Plasma," $19^{\text {th }}$ International Conference on Atomic Physics, Rio de Janero,Brazil, (7/29/04).

"Imaging an Ultracold Neutral Plasma," 2000 Annual Meeting of the Division of Atomic, Molecular, and Optical Physics of the American Physical Society, Tucson, Arizona, (5/28/04).

"Ultracold Neutral Plasmas," Lectures at the International Workshop and Seminar on Rydberg Physics, Dresden, Germany, (4-5/04).

"Imaging an Ultracold Neutral Plasma," Harvard/MIT Center for Ultracold Atoms Seminar, Cambridge, MA (4/22/04).

"Collisions in Ultracold Neutral Plasmas," XXIII International Conference on Photonic, Electronic, and Atomic Collisions, Stockholm, Sweden (7/22/03).

Fundamental interest in ultracold neutral plasmas stems from a range of phenomena in the ultracold regime. It is possible to form strongly coupled neutral plasmas - systems in which the electrical interaction energy between the charged particles exceeds the average kinetic energy. This reverses the traditional energy hierarchy that underlies our normal understanding of plasmas based on concepts such as Debye screening and hydrodynamics. Strongly coupled plasmas appear in exotic environments that are hard to probe, such as white dwarf stars and inertial confinement fusion systems. Non-neutral plasmas of positively charged ions and dusty plasmas of highly charged macroscopic particles that are cooled into this regime undergo phase transitions from gaseous states to beautiful solid-like Wigner crystals that minimize the particles' interaction energy. The neutral systems with which I work have proven to be just as rich in new physics. The presence of electrons, in addition to ions, introduces many more phenomena such as recombination and an assortment of collective particle motions. Recombination in these systems resembles the methods to produce cold antihydrogen atoms at CERN. Improved understanding of the comparatively simple experiments I perform will help our interpretation of more complex anti-matter work.

DOE funding for this work is now ending. The impression I received from program officials was that the research was too basic to fit into the portfolio of the Office of Fusion Energy Sciences, so I have not sought out additional funding from this resource. The initial support of the Junior Faculty Program was crucial to the success of this research, however, and for that I am indebted. I expect a large volume of experimental publications during the next year as we exploit the optical imaging probe. This will help us understand the complicated equilibration process and early dynamics of ultracold plasmas. Armed with this information we will pursue our longer range goal of laser-cooling an ultracold neutral plasma. This should lead to drastically colder systems, and would represent a new mode of plasma confinement. 


\title{
Using Absorption Imaging to Study Ion Dynamics in an Ultracold Neutral Plasma
}

\author{
C. E. Simien, Y. C. Chen, P. Gupta, S. Laha, Y. N. Martinez, P. G. Mickelson, S. B. Nagel, and T. C. Killian \\ Department of Physics and Astronomy and Rice Quantum Institute, Rice University, Houston, Texas 77251, USA
}

(Received 17 September 2003; published 6 April 2004)

\begin{abstract}
We report optical absorption imaging of ultracold neutral strontium plasmas. The ion absorption spectrum determined from the images is Doppler broadened and thus provides a quantitative measure of the ion kinetic energy. For the particular plasma conditions studied, ions heat rapidly as they equilibrate during the first $250 \mathrm{~ns}$ after plasma formation. Equilibration leaves ions on the border between the weakly coupled gaseous and strongly coupled liquid states. On a longer time scale of microseconds, pressure exerted by the trapped electron gas accelerates the ions radially.
\end{abstract}

DOI: 10.1103/PhysRevLett.92.143001

PACS numbers: $32.80 . P \mathrm{j}, 52.27 . \mathrm{Gr}$

Plasma physics traditionally studies systems with temperatures of thousands of kelvin or greater because collisional ionization of atoms requires kinetic energies on this scale. Ultracold neutral plasmas, created by photoionizing laser-cooled atoms, access an exotic regime in which particle energies can be on the order of $1 \mathrm{~K}$.

Fundamental interest in ultracold neutral plasmas stems from a range of phenomena in the ultracold regime. Recent experiments studied plasma creation [1], collective modes [2], and recombination into highly excited Rydberg atomic states [3]. Recombination in these systems resembles methods used to produce cold antihydrogen from trapped positrons and antiprotons [4,5]. The spontaneous evolution of a dense, ultracold gas of Rydberg atoms into an ultracold plasma was also investigated [6].

A series of theory papers [7-11] explored issues surrounding thermalization and recombination in ultracold neutral plasmas when both electrons and ions are near or in the strongly coupled regime [12]. In strongly coupled systems the electrical interaction energy between charged particles exceeds the average kinetic energy, reversing the energy hierarchy found in traditional plasmas.

We report results with a new probe of ultracold plasmas: absorption imaging of a strontium plasma using the $\mathrm{Sr}^{+}{ }^{2} S_{1 / 2}-{ }^{2} P_{1 / 2}$ transition at $422 \mathrm{~nm}(\Gamma / 2 \pi=$ $22 \mathrm{MHz}$ ). This technique provides in situ, nondestructive measurements and offers excellent spatial, temporal, and spectral resolution. We describe the use of this probe to study ion-ion equilibration and expansion of the plasma during the first few microseconds after photoionization, but we emphasize its potential to study a host of phenomena such as ion collective modes [13], shock waves [10], recombination, and particle-particle spatial correlations [14].

The production of the ultracold neutral plasma starts with strontium atoms that are cooled and confined in a magneto-optical trap (MOT) [15] (Fig. 1). The neutral atom cloud has a temperature of about $20 \mathrm{mK}$ and a density distribution $n(r)=n_{0} \exp \left(-r^{2} / 2 \sigma^{2}\right)$, with $\sigma=1 \mathrm{~mm}$ and $n_{0}=(4 \pm 2) \times 10^{10} \mathrm{~cm}^{-3}$. The number of trapped atoms is $(6 \pm 1) \times 10^{8}$.

The MOT magnets are then turned off, and atoms that are excited to the ${ }^{1} P_{1}$ level by the MOT lasers are ionized with photons from a $10 \mathrm{~ns}$ pulsed dye laser. The pulsed laser wavelength is tuned just above the ionization continuum. Because of the small electron-to-ion mass ratio, the initial electron kinetic energy $\left(E_{e}\right)$ approximately equals the difference between the photon energy and the ionization potential. $E_{e} / k_{B}$ can be as low as the bandwidth of the ionizing laser, which is $\sim 100 \mathrm{mK}$. The initial kinetic energy for the resulting singly charged, electronic ground state ions is close to that of the original neutral atoms. As we will discuss below, the resulting nonequilibrium plasma evolves rapidly. Up to $12 \% \pm$ $1 \%$ of the neutral atoms are ionized, producing plasmas with a peak density of $(5 \pm 3) \times 10^{9} \mathrm{~cm}^{-3}$.

Immediately after photoionization, the charge distribution is neutral everywhere. Because of the kinetic energy of the electrons, the electron cloud expands on the time scale of the inverse electron plasma frequency $\tau_{e}=\omega_{p e}^{-1}=\sqrt{m_{e} \varepsilon_{0} / n_{e} e^{2}}<1 \mathrm{~ns}$, where $m_{e}, n_{e}$, and $e$ are the electron mass, density, and charge. On this time scale ions are essentially immobile. The resulting charge

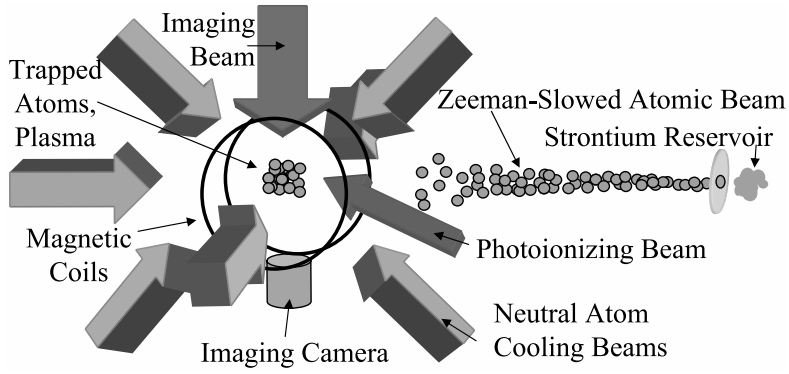

FIG. 1. Experimental schematic. Neutral atoms are laser cooled and trapped in a magneto-optical trap operating on the ${ }^{1} S_{0}-{ }^{1} P_{1}$ transition at $461 \mathrm{~nm} .{ }^{1} P_{1}$ atoms are ionized by photons from a laser at $\sim 412 \mathrm{~nm}$. Ions are imaged using the ${ }^{2} S_{1 / 2}-{ }^{2} P_{1 / 2}$ transition at $422 \mathrm{~nm}$. 
imbalance creates a Coulomb potential energy well that traps all but a small fraction $(<5 \%)$ of the electrons. Simulations [1] show that electrons escape mostly from the edges of the spatial distribution, and the center of the cloud is well described as a neutral plasma [16]. The diagnostic in previous experiments was detection of electrons and ions after they had left the plasma.

Spectroscopic diagnostics are ubiquitous in plasma experiments, and some even provide spatial information, such as spatially resolved laser induced fluorescence $[14,17]$. The absorption imaging reported here is particularly well adapted for small, cold, relatively dilute plasmas that evolve very quickly. It is also a powerful technique for studying laser-cooled and trapped neutral atoms [18]. A collimated laser beam, tuned near resonance with the principle transition in the ions (Fig. 1), illuminates the plasma and falls on an image intensified CCD camera. Following Beer's law, the optical depth (OD) is defined in terms of the image intensity without $\left(I_{\text {background }}\right)$ and with $\left(I_{\text {plasma }}\right)$ the plasma present,

$$
\begin{aligned}
\mathrm{OD}(x, y) & =\ln \left[I_{\text {background }}(x, y) / I_{\text {plasma }}(x, y)\right] \\
& =\alpha(\nu) \int_{-\infty}^{\infty} d z n_{i}(x, y, z) \\
& =\frac{n_{0 i} \alpha(\nu)}{\sqrt{2 \pi} \sigma_{z}} e^{-x^{2} / 2 \sigma_{x}^{2}-y^{2} / 2 \sigma_{y}^{2}}
\end{aligned}
$$

where $n_{0 i}$ is the peak ion density, and $\alpha(\nu)$ is the absorption cross section at the image beam frequency, $\nu$. We have inserted a Gaussian density distribution for the ions, which leads to the function used to fit the data.

Figure 2 shows a typical absorption image. The intensity of the probe beam is $200 \mu \mathrm{W} / \mathrm{cm}^{2}$, which is much less than the saturation intensity of the transition $\left(38 \mathrm{~mW} / \mathrm{cm}^{2}\right)$. The spatial resolution of typical images is $100 \mu \mathrm{m}$, limited by pixel averaging performed to improve the signal-to-noise ratio of the images.

To study the time evolution of the plasma, we vary the delay between the formation of the plasma and image exposure $\left(t_{\text {delay }}\right)$ with $10 \mathrm{~ns}$ accuracy. The minimum camera exposure gate width is $50 \mathrm{~ns}$. For the shortest exposure times, which we use for the best time resolution at very
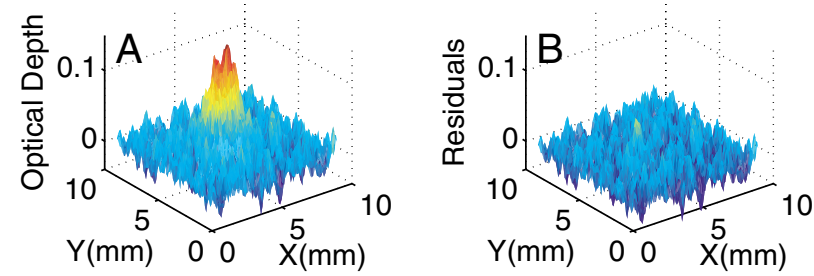

FIG. 2 (color online). Optical depth of an ultracold neutral plasma. (a) The delay between the formation of the plasma and image exposure is $100 \mathrm{~ns}$, and the initial peak density is $n_{0 i}=$ $5 \times 10^{9} \mathrm{~cm}^{-3}$. (b) Residuals of a fit to a two-dimensional Gaussian profile are close to the photon shot noise. short $t_{\text {delay }}$, we typically average about 60 ionizing laser shots. For longer delay times we use longer image exposure times of up to $800 \mathrm{~ns}$, and decrease the number of accumulations to keep the total number of photons detected approximately constant. The repetition rate for ionization and image recording is about 5 times per second.

Plotting the peak optical depth as a function of image laser frequency provides the absorption spectrum of the ions (Fig. 3). The imaging laser linewidth of about $5 \mathrm{MHz}$ is negligible on the scale of the natural linewidth of the transition. As described below, additional broadening of the absorption spectrum provides a wealth of information on the plasma dynamics.

Here we describe experiments using the time evolution of the absorption spectrum to study ion dynamics for a plasma with $N_{i}=7 \times 10^{7}$ ions, initial peak density for ions and electrons of $n_{0 i} \approx n_{0 e}=(5 \pm 3) \times 10^{9} \mathrm{~cm}^{-3}$, and $E_{e} / k_{B}=(68 \pm 5) \mathrm{K}$. We chose a relatively large $E_{e}$ in order to avoid complications that arise when the electron Coulomb coupling parameter $\left(\Gamma_{e}=e^{2} / 4 \pi \varepsilon_{0} a k_{B} T_{e}\right)$ approaches or initially exceeds unity, such as screening of the ion interaction [8], and rapid collisional recombination and heating of the electrons [8-11]. Here, $a=$ $\left(4 \pi n_{0 e} / 3\right)^{-1 / 3}$ is the Wigner-Seitz radius, and $T_{e}=$ $\frac{2}{3} E_{e} / k_{B}$ is the electron temperature as set by the wavelength of the ionizing laser. For this sample $\Gamma_{e}=0.1$.

The observed spectral linewidths are significantly broader than the natural linewidth of the transition. Quasistatic Stark broadening by ions is negligible because we are dealing with deeply bound ionic levels that have a small quadratic Stark shift in the typical plasma electric field $\left(e / 4 \pi \varepsilon_{0} a^{2} \sim 1 \mathrm{~V} / \mathrm{cm}\right)$. An impact approximation $[19,20]$ suggests that broadening due to electron collisions is on the order of $1 \mathrm{MHz}$ and can also be neglected. The

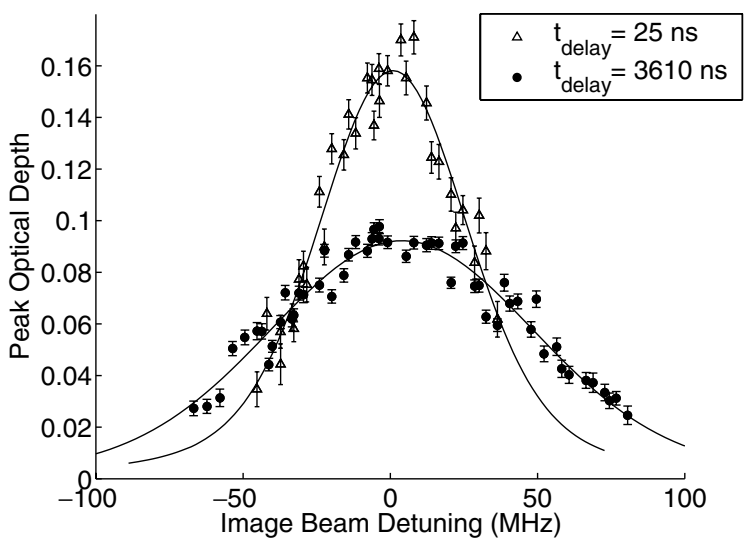

FIG. 3. Absorption spectra of ultracold neutral plasmas. We plot the peak optical depth derived from fits to data such as Fig. 2. The frequency is with respect to a Doppler-free absorption feature in a strontium discharge cell. Both spectra correspond to $E_{e} / k_{B}=68 \mathrm{~K}$ and the same initial peak plasma density of $n_{0 i}=5 \times 10^{9} \mathrm{~cm}^{-3}$. Data are fit with Voigt profiles, and the increase in linewidth for longer $t_{\text {delay }}$ is clear. 
dominant contribution to the linewidth beyond the natural width is Doppler broadening, which makes the spectrum a very accurate probe of the ion velocity distribution. From the fit of each spectrum to a Voigt profile using a Lorentzian width equal to the natural width, we extract the rms Doppler broadening, $\sigma_{D}=\sqrt{k_{B} T_{i} / m_{i}} / \lambda$, where $m_{i}$ is the ion mass, and $\lambda$ is the wavelength of the transition. This provides the ion temperature $\left(T_{i}\right)$ as a function of time, as shown in Fig. 4.

The temperature increases rapidly for $t_{\text {delay }}<250 \mathrm{~ns}$. Two pieces of information imply that this is thermalization of ions with themselves after creation in a spatially disordered state. The time scale is on the order of the inverse plasma frequency of ions, $\tau_{i}=\omega_{p i}^{-1}=$ $\sqrt{m_{i} \varepsilon_{0} / n_{0 i} e^{2}}=100 \mathrm{~ns}$, which is the time scale on which ions respond to perturbations from their equilibrium spatial distribution. The temperature of $T_{i}=1.4 \pm 0.1 \mathrm{~K}$ derived from the Doppler width at $250 \mathrm{~ns}$ is also on the order of the amount of potential energy inherent in the initial disorder of the ions. A simple estimate implies there is $e^{2} / 4 \pi \varepsilon_{0} a k_{B} \approx 5 \mathrm{~K}$ of potential energy that will be redistributed during thermalization.

This thermalization has been modeled with molecular dynamics simulations [7-9], and the experimental data are in general agreement with the theory. The accuracy of the imaging probe will enable a detailed comparison of measurement and theory for the time dependence of the ion temperature.

The final temperature reached can be quantitatively compared with an expression derived to describe disorder-induced heating in ultracold neutral plasmas [7]. Assuming complete initial disorder and incorporating

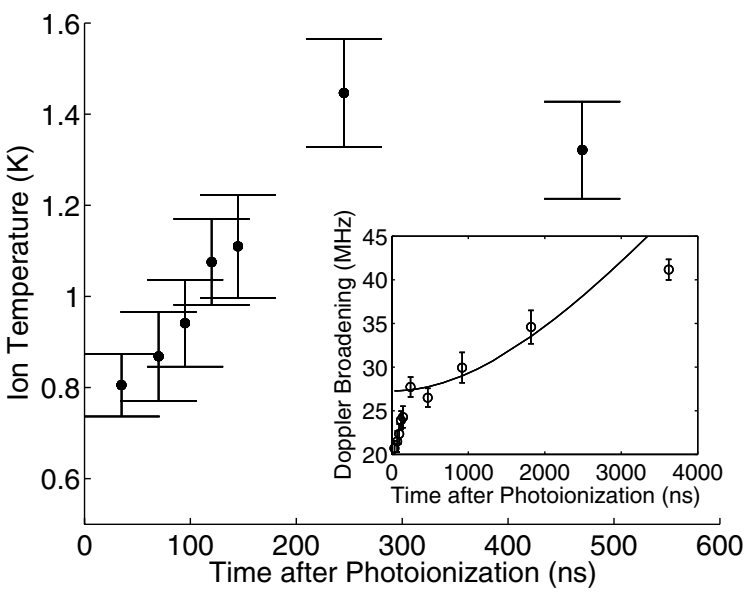

FIG. 4. Ion dynamics determined from the spectral width. Error bars are two sigma uncertainties from Voigt profile fits to absorption spectra. The time after photoionization corresponds to the timing of the center of the camera exposure gate. Data show disorder-induced heating of the ions as the ions equilibrate during $t_{\text {delay }}<250 \mathrm{~ns}$. (Inset) For longer times, the rms Doppler broadening of the spectrum continues to increase as ions accelerate radially due to electron pressure. This process is described by a hydrodynamic model with no free parameters. screening effects of electrons through a Yukawa potential [21], the equilibrium ion temperature is predicted to be

$$
T_{i}=\frac{2}{3} \frac{e^{2}}{4 \pi \varepsilon_{0} a k_{B}}\left|\tilde{U}+\frac{\kappa}{2}\right|
$$

Here $\quad \kappa=a / \lambda_{D}=\sqrt{3 \Gamma_{e}}=0.55$, where $\lambda_{D}=$ $\left(\varepsilon_{0} k_{B} T_{e} / n_{0 e} e^{2}\right)^{1 / 2}=7 \mu \mathrm{m}$ is the Debye length. The quantity $\tilde{U} \equiv U /\left(N_{i} e^{2} / 4 \pi \varepsilon_{0} a\right)$ is the potential energy per particle in units of $e^{2} / 4 \pi \varepsilon_{0} a$. It has not been calculated for ultracold neutral plasmas specifically, so we use molecular dynamics simulations intended for dusty plasmas and colloidal suspensions [22]. The systems are similar (massive, cold component immersed in a light, weakly coupled neutralizing background), but this approximation merits further investigation. Combining results from [22] with measured experimental parameters implies $\tilde{U}=-0.73$. Equation (2) then predicts an equilibrium ion temperature of $1.4 \mathrm{~K}$, in agreement with the measured value.

We now address the level of Coulomb coupling for the ions. The measured temperature and peak density imply $\Gamma_{i}=3 \pm 1$ for the thermalized ion cloud at $t_{\text {delay }}=$ $250 \mathrm{~ns}$. For a system of charges embedded in a uniform neutralizing background, formally called a onecomponent plasma [23], local spatial correlations characteristic of a strongly coupled fluid appear for $\Gamma \geq 2$. For Debye shielded particles, such as ions studied here, screening reduces correlations. This effect is approximately incorporated by using the effective coupling constant $\Gamma^{*}=\Gamma \mathrm{e}^{-\kappa}[8,22]$. At $250 \mathrm{~ns}, \Gamma_{i}^{*}=2 \pm 1$, and the ions are just on the edge of the strongly coupled fluid phase. Perhaps other initial experimental parameters, or laser cooling of ions $[8,24]$, will lead to more strongly coupled systems, although $\Gamma_{i}^{*}=2$ already puts the experiment in an interesting regime.

For $t_{\text {delay }}>250 \mathrm{~ns}$, the spectral width continues to increase, but at a slower rate, as shown in the inset of Fig. 4. This slow increase results from outward radial acceleration of the ions caused by pressure exerted by the gas of trapped electrons. This was studied experimentally in [2] and theoretically by a variety of means in [10]. The experiments measured the final velocity that the ions acquired, which was approximately $\sqrt{E_{e} / m_{i}}$. Here we observe ion dynamics at much earlier times during the acceleration phase.

A hydrodynamic treatment [2] predicts a force per ion of

$$
\bar{F}=-\frac{\bar{\nabla}\left[n_{e}(r) k_{B} T_{e}\right]}{n_{i}(r)}=\hat{r} \frac{r k_{B} T_{e}}{\sigma_{i}^{2}}
$$

where the electron and ion density distributions are $n_{e}(r) \approx n_{i}(r)=n_{0 i} \exp \left(-r^{2} / 2 \sigma_{i}^{2}\right)$. We approximate the rms size $\left(\sigma_{i}\right)$ as the mean of the observed sizes $\sigma_{x}$ and $\sigma_{y}$, and we assume thermal equilibrium for the electrons throughout the cloud [10]. This force leads to a radial 
expansion velocity for the ions, $v_{r}(r)$, that correlates with position and increases linearly with time. This does not represent an increase in the random thermal velocity spread or temperature of the ions. Because of the large mass difference, thermalization of ions and electrons [8] is slow and occurs on a millisecond time scale. The increase in Doppler broadening due to thermalization is approximately 1 order of magnitude smaller than what is observed.

Using the dynamics implied by Eq. (3), the evolution of the Doppler broadening can be calculated. The mean squared velocity component along the imaging laser is

$$
\left\langle\boldsymbol{v}_{z}^{2}\right\rangle=\int d^{3} r d v_{T} \frac{n_{i}(r)}{N_{i}} P\left(\boldsymbol{v}_{T}\right)\left[\boldsymbol{v}_{T}+\boldsymbol{v}_{r}(r) \cos \theta\right]^{2},
$$

where $P\left(v_{T}\right)$ is the thermal distribution of $v_{z}$ for $T_{i}=$ $1.4 \mathrm{~K}$. The resulting theoretical Doppler broadening $\sqrt{\left\langle\boldsymbol{v}_{z}^{2}\right\rangle} / \lambda$, plotted in Fig. 4, reproduces the data accurately. Ion acceleration is thus an excellent diagnostic of the electron temperature. This will be of great value in future studies because the temperature is predicted to evolve in a complicated fashion for higher initial $\Gamma_{e}$ due to recombination and disorder-induced heating of the electrons [811]. One also expects that there will be a cooling effect at longer $t_{\text {delay }}$ due to plasma expansion and evaporative cooling [10]. The small discrepancy between theory and data for long $t_{\text {delay }}$ in Fig. 4 may indicate the onset of this cooling, although plasma expansion is small on the time scale of these observations. For the maximum $t_{\text {delay }}$ and typical $v_{r} \approx 15 \mathrm{~m} / \mathrm{s}, v_{r} t_{\text {delay }} \approx 50 \mu \mathrm{m}$. This small increase in size is observed in the images (Fig. 2).

The initial study using absorption imaging of an ultracold neutral plasma has probed ion dynamics in the first few microseconds after photoionization. It revealed disorder-induced heating that was predicted in [7] and showed that the ions equilibrate on the boundary of the gas-liquid transition. Acceleration of ions due to electron pressure was also evident and can be used to monitor the electron temperature.

Many future experiments suggest themselves, such as investigating dynamics when the initial electron Coulomb coupling parameter is large and recombination and disorder-induced electron heating are expected to dominate the plasma evolution. Detailed study of ion and electron thermalization at the border of the strongly coupled regime is also possible. Improvements in imaging optics will increase the image signal-to-noise ratio and allow the study of features on the ion density distribution with $\sim 10 \mu \mathrm{m}$ experimental resolution.

We acknowledge helpful discussions with T. M. O'Neil, C. H. Greene, R. Santra, M. S. Murillo, F. Robicheaux, S. Mazevet, and J. P. Colgan. This research was supported by the Department of Energy Office of Fusion Energy Sciences, Office for Naval Research, Research Cor- poration, Alfred P. Sloan Foundation, and David and Lucille Packard Foundation.

[1] T. C. Killian, S. Kulin, S. D. Bergeson, L. A. Orozco, C. Orzel, and S. L. Rolston, Phys. Rev. Lett. 83, 4776 (1999).

[2] S. Kulin, T. C. Killian, S. D. Bergeson, and S. L. Rolston, Phys. Rev. Lett. 85, 318 (2000).

[3] T. C. Killian, M. J. Lim, S. Kulin, R. Dumke, S. D. Bergeson, and S. L. Rolston, Phys. Rev. Lett. 86, 3759 (2001).

[4] M. Amoretti et al., Nature (London) 419, 456 (2002).

[5] G. Gabrielse et al., Phys. Rev. Lett. 89, 213401 (2002).

[6] M. P. Robinson, B. L. Tolra, M.W. Noel, T. F. Gallagher, and P. Pillet, Phys. Rev. Lett. 85, 4466 (2000).

[7] M. S. Murillo, Phys. Rev. Lett. 87, 115003 (2001).

[8] S. G. Kluzmin and T. M. O’Neil, Phys. Plasmas 9, 3743 (2002).

[9] S. Mazevet, L. A. Collins, and J. D. Kress, Phys. Rev. Lett. 88, 055001 (2002).

[10] F. Robicheaux and J. D. Hanson, Phys. Plasmas 10, 2217 (2003).

[11] A. N. Tkachev and S. I. Yakovlenko, Quantum Electron. 30, 1077 (2000).

[12] S. Ichimuru, Rev. Mod. Phys. 54, 1017 (1982).

[13] M. S. Murillo, Phys. Rev. Lett. 85, 2514 (2000).

[14] T. B. Mitchell, J. J. Bollinger, X. P. Huang, W. M. Itano, and D. H. E. Dubin, Phys. Plasmas 6, 1751 (1999).

[15] S. B. Nagel, C. E. Simien, S. Laha, P. Gupta, V. S. Ashoka, and T. C. Killian, Phys. Rev. A 67, 011401 (2003).

[16] The approximate excess ion density is $3 n_{e}\left(\lambda_{D} / \sigma\right)^{2} \ll n_{e}$, where $\lambda_{D}$ is the Debye screening length.

[17] F. M. Levinton and F. Trintchouk, Rev. Sci. Instrum. 72, 898 (2001).

[18] H. J. Metcalf and P. van der Straten, Laser Cooling and Trapping (Springer-Verlag, New York, 1999).

[19] H. R. Griem, Spectral Line Broadening by Plasmas (Academic Press, New York, 1974).

[20] To estimate electron impact broadening we evaluated an expression for hydrogenic ions using an effective principle quantum number calculated from the quantum defects of the strontium ion $\left(\delta_{s} \approx 2.7\right.$ and $\left.\delta_{p} \approx 2.3\right)$. An estimate using more accurate $\mathrm{Sr}^{+}$wave functions and including $s, p$, and $d$ partial waves yields $40 \mathrm{kHz}$ for the broadening [C. H. Greene and R. Santra (private communication)].

[21] As the number of electrons per Debye sphere $\left(\kappa^{-3}\right)$ approaches unity, the Yukawa interaction ceases to accurately describe ion-ion interactions. For the strontium plasma $\left(\kappa^{-3}=6\right)$ the model is reasonably valid.

[22] R. T. Farouki and S. Hamaguchi, J. Chem. Phys. 101, 9885 (1994).

[23] D. H. E. Dubin and T. M. O’Neil, Rev. Mod. Phys. 71, 87 (1999).

[24] T. C. Killian, V. S. Ashoka, P. Gupta, S. Laha, S. B. Nagel, C. E. Simien, S. Kulin, S. L. Rolston, and S. D. Bergeson, J. Phys. A 36, 6077 (2003). 


\title{
Electron Screening and Kinetic Energy Oscillations in a Strongly Coupled Plasma
}

\author{
Y. C. Chen, C. E. Simien, S. Laha, P. Gupta, Y. N. Martinez, P. G. Mickelson, S. B. Nagel, and T. C. Killian \\ Rice University, Department of Physics and Astronomy and Rice Quantum Institute, Houston, Texas, 77005
}

(Dated: August 9, 2004)

\begin{abstract}
We study equilibration of strongly coupled ions in an ultracold neutral plasma produced by photoionizing laser-cooled and trapped atoms. By varying the electron temperature, we show that electron screening modifies the equilibrium ion temperature. Even with few electrons in a Debye sphere, the screening is well-described by a model using a Yukawa ion-ion potential. We also observe damped oscillations of the ion kinetic energy that are a unique feature of equilibration of a strongly coupled plasma.
\end{abstract}

A plasma becomes strongly coupled when the electrical interaction energy between the charged particles exceeds the thermal energy. Strong coupling is observed in dense astrophysical environments [1], such as the interiors of gas giant planets, and in laser-cooled ions [2], dusty plasmas [3], and solid targets [4] and clusters [5] irradiated by pulsed high-intensity lasers. Equilibration in these systems is of particular interest because it involves the establishment of spatial correlations between particles.

There has been significant theoretical study of equilibration of strongly coupled plasmas [6-12], especially in the context of plasmas produced with high-intensity laser. In addition to the fundamental interest, this problem challenges computational resources and techniques. Experimental results have been lacking, however, because of the fast timescales involved and limited diagnostics.

Ultracold neutral plasmas [13], produced by photoionizing clouds of laser-cooled and trapped atoms, are ideal for experimental studies. The equilibration of the plasma is relatively slow $(\sim 100 \mathrm{~ns})$ due to lower plasma density. Ultracold neutral plasmas also offer a high level of control and diagnostics. By varying laser intensities and wavelengths it is possible to accurately set the initial density and energy of the system. Optical imaging [14] provides an in situ probe of plasma properties with excellent spatial, temporal, and spectral resolution.

In this paper, we explore ion equilibration during the first microsecond after the plasma is created. The density sets the time and energy scale for equilibration, but electron screening effects are evident. Even when the number of electrons per Debye sphere is close to unity, the equilibration temperature of the ions agrees with a model [15] that uses a Yukawa ion-ion potential.

We also observed oscillations of the ion kinetic energy. For many years, this phenomenon has been the subject of intense study through analytic calculations [7] and simulations [6, 8-12] of one-component strongly coupled plasmas, but it has not previously been observed experimentally. The oscillations and their damping reflect universal dynamics of a Coulomb system with spatial correlations.

Details on laser cooling, plasma formation and imaging are given in $[14,16]$. The experiment starts with strontium atoms that are cooled and trapped in a magnetooptical trap (MOT). The neutral atom cloud is characterized by a temperature of about $10 \mathrm{mK}$, typically $2 \times 10^{8}$ atoms, and a Gaussian density distribution. We vary the atom density by changing the MOT parameters, or by turning the MOT off and releasing the atoms in a ballistic expansion. Up to $30 \%$ of the neutral atoms are then ionized with one photon from the cooling laser and one photon from a pulsed dye laser. The ion density distribution equals the atom distribution at the time of photoionization and is given by $n_{i}(r)=n_{0 i} \exp \left(-r^{2} / 2 \sigma^{2}\right)$, with $\sigma$ from 0.6 to $1 \mathrm{~mm}$ and $n_{0 i}$ from $2 \times 10^{9}$ to $1.4 \times 10^{10} \mathrm{~cm}^{-3}$. The electron density, $n_{e}(\mathbf{r})$, closely follows the ion density, and the initial electron temperature is given by $T_{e}=2 E_{e} / 3 k_{B}$, where $E_{e}$ is the detuning of the pulsed laser above the ionization threshold.

At an adjustable delay time $\left(t_{\text {delay }}\right)$ after photoionization, a collimated laser beam, tuned near resonance with the $\mathrm{Sr}^{+}{ }^{2} S_{1 / 2}-{ }^{2} P_{1 / 2}$ transition at $422 \mathrm{~nm}$, illuminates the plasma and falls on an intensified CCD camera. By varying $t_{\text {delay }}$, we study the evolution of the plasma after formation.

The optical depth $(O D)$ for a laser propagating along the $\mathrm{z}$ axis is defined by $O D(x, y)=$ $\log \left[I_{\text {background }}(x, y) / I_{\text {image }}(x, y)\right]$, where $I_{\text {background }}$ and $I_{\text {image }}$ are laser intensities without and with the plasma present. By integrating the optical depth over $\mathrm{x}$ and $\mathrm{y}$ for images taken at different image beam frequencies, $\nu$, we obtain the absorption spectrum

$$
\begin{aligned}
S(\nu) & =\int \mathrm{d} x \mathrm{~d} y O D(x, y)=\int \mathrm{d}^{3} r n_{i}(r) \alpha\left[\nu, T_{i}(r)\right] \\
& =N_{i} \alpha\left(\nu, T_{i, \text { eff }}\right) .
\end{aligned}
$$

We have used Beer's law to relate $S(\nu)$ to the absorption cross section, $\alpha\left[\nu, T_{i}(r)\right]$, and the ion density. The absorption cross section is a function of temperature due to the Doppler broadening, and since we expect the temperature to vary with density, we allow $\alpha$ to vary with position. The absorption cross section has a Voigt profile resulting from the convolution of the Gaussian Doppler broadening with a Lorentzian with width $\gamma=\gamma_{0}+\gamma_{\text {laser }}$. Here, $\gamma_{0}=22 \mathrm{MHz}$ is the natural width of the transition, and $\gamma_{\text {laser }}=(10 \pm 2) \mathrm{MHz}$ is the measured linewidth of the laser.

As shown in the second line of Eq.1, we fit the spectrum to a single Voigt profile with effective temperature $T_{i, \text { eff }}$. $N_{i}$ is the number of ions. We will argue below that $T_{i, \text { ef } f}$ is a good approximation of the average ion temperature in the plasma. Fig 1(a) shows the evolution of $T_{i, e f f}$ for 

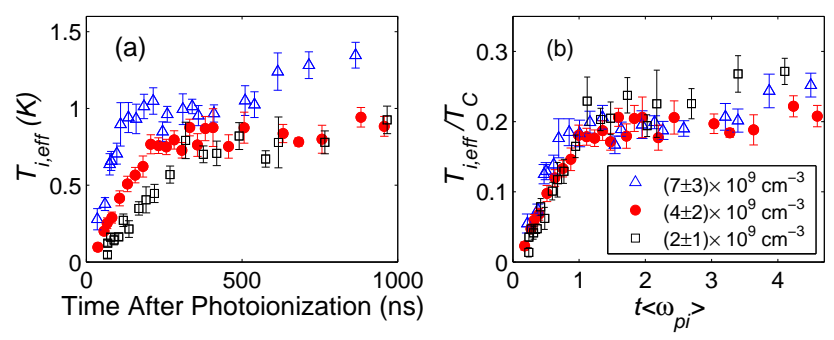

FIG. 1: The effective ion temperature, $T_{i, e f f}$, versus time after photoionization for initial electron temperature of $T_{e}=$ $40 \mathrm{~K}$ and various plasma densities. (A) The data is plotted on absolute temperature and time scales. (B) The time is scaled by the inverse of the average plasma period, and $T_{i, \text { ef } f}$ is scaled by $T_{C}$.

three different densities. The rapid increase in the temperature for $t_{\text {delay }}<300 \mathrm{~ns}$ is due to disorder-induced heating. This originates from conversion of Coluomb potential energy into kinetic energy as the ions evolve from a completely disordered state to one with some degree of spatial correlations. This was predicted in [15], and observed in numerical simulations $[12,17,18]$ and the first experimental studies with optical imaging [14].

The time scale of the heating is the inverse ion plasma frequency $\omega_{p i}^{-1}=\sqrt{m_{i} \varepsilon_{0} / n_{i} e^{2}} \approx 100 \mathrm{~ns}$. The energy scale is $T_{C}=e^{2} / 4 \pi \varepsilon_{0} a k_{B} \approx 5 \mathrm{~K}$, where $a=\left(4 \pi n_{i} / 3\right)^{-1 / 3}$ is the Wigner-Seitz radius. Fig 1(b) shows data with time scaled by the average inverse plasma frequency and temperature scaled by $T_{C}$. The three curves coincide very well in the time axis, but show slight deviation in the temperature axis. The deviation indicates the effects of electron screening of the ion-ion interaction.

A detailed expression for the equilibrium ion temperature, including screening effects, was derived in [15]. Assuming complete disorder and stationary ions at $t_{\text {delay }}=$ 0 , and incorporating electron screening through a Yukawa ion-ion potential $[19,20]$,

$$
T_{i}=\frac{2}{3} \frac{e^{2}}{4 \pi \varepsilon_{0} a k_{B}}|\tilde{U}+\kappa / 2| .
$$

Here, $\kappa=a / \lambda_{D}$, where $\lambda_{D}=\left(\varepsilon_{0} k_{B} T_{e} / n_{i} e^{2}\right)^{1 / 2}$ is the Debye length. The quantity $\tilde{U} \equiv \frac{U}{N_{i} e^{2} / 4 \pi \varepsilon_{0} a}$ is the potential energy per particle in units of $e^{2} / 4 \pi \varepsilon_{0} a$, and it has been tabulated in [20]. Equation 2 is complicated by the fact that $\tilde{U}$ is a function of $n_{i}$ and $T_{i}$. From the table of $\tilde{U}$ and using an iterative numerical routine, $T_{i}$ can be obtained for a given $n_{i}$ and $T_{e}$.

The effects of electron screening are quantified by the factor $|\tilde{U}+\kappa / 2|$. Colder electron temperature and higher density leads to a smaller Debye length and greater screening of the ion-ion potential. This decreases the potential energy in the initial system, and thus, compared to a system in which there is no screening, decreases the equilibration temperature reached. This effect is evident in Fig. 2, which shows data from three different

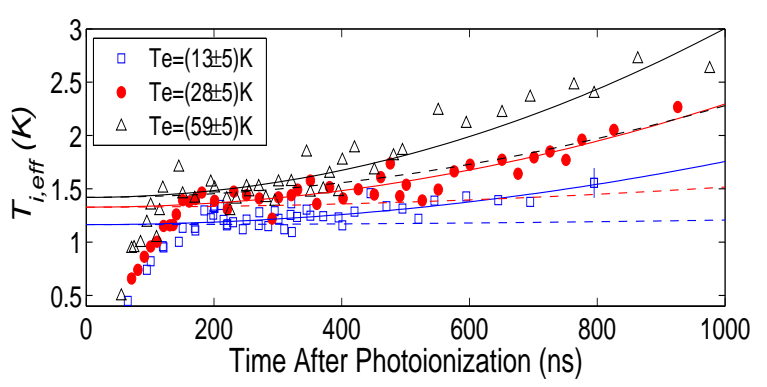

FIG. 2: Effective ion temperature versus time after photoionization for various electron temperatures. The peak ion density for all data is $n_{0 i}=(1.4 \pm 0.5) \times 10^{10} \mathrm{~cm}^{-3}$. The solid curve is a fit of the data based on Eq. 3. The fit determines the equilibrium $T_{i, a v e}$ and $T_{e}$. The fit $T_{e},(80 \pm 5),(63 \pm 6)$, and $(48 \pm 6) \mathrm{K}$ are higher than the expected initial $T_{e}$, indicating electron heating. The dashed curves show the increase in $T_{i, e f f}$ due to radial acceleration expected for $T_{e}=2 E_{e} / 3 k_{B}$. A typical data error bar is shown.

initial electron energies, but the same ion density distribution. To quantitatively compare the data in Figs. 1 and 2 with Eq. 2 we must account for two factors. The first is the effect of the inhomogeneous density distribution. We expect the ion temperature to vary with density because global thermal equilibrium occurs on a hydrodynamic time scale, $\sigma / v$, which is on the order of tens of $\mu \mathrm{s}$, where $v$ is the ion acoustic wave velocity. Local thermal equilibration occurs on a much faster time scale, $\sim \omega_{p i}^{-1}[21]$. If we assume a temperature distribution given by Eq. 2, numerical simulations [22] show that $T_{i, \text { eff }}=(0.95 \pm 0.05) T_{i, \text { ave }}$. Here $T_{i, \text { ave }}$ is the average ion temperature found by averaging Eq. 2 over the density profile.

To extract the average ion temperature, we must also account for acceleration of the ions by the electron pressure. This was studied experimentally in $[14,23]$ and theoretically in $[24,25]$, and it is evident in the continuing increase in $T_{i, e f f}$ in Figs. 1 and 2 for $t_{\text {delay }}>500$ ns. This acceleration will lead to expansion of the plasma after a time on the order of $10 \mu \mathrm{s}$, and although it is not a heating effect, it contributes to the Doppler width of the spectrum. Numerical analysis [22], assuming a temperature distribution given by Eq. 2, shows that the effect of plasma expansion on the effective ion temperature can be approximated by,

$$
T_{i, \text { eff }}\left(t_{\text {delay }}\right)=C T_{i, \text { ave }}\left[1+1 / C\left(t_{\text {delay }} / t_{\text {exp }}\right)^{2}\right] .
$$

where $C=(0.95 \pm 0.05)$ is a constant and $t_{\exp }$ is the time scale when the Doppler broadening contributed by plasma expansion is comparable to the thermal Doppler broadening. It is given by,

$$
t_{e x p}=\sigma \sqrt{m_{i} / k_{B} T_{e}} \sqrt{T_{i, a v e} / T_{e}}
$$

This quantifies our earlier statement that $T_{i, e f f}$ is a good measure of the average ion temperature for $t_{\text {delay }}>\omega_{p i}^{-1}$. 
Because we want to check the accuracy of Eq. 2, and we assume this equation in our analysis, strictly speaking, our strongest conclusion can be that our data are consistent with Eq. 2. However, we found [22] that Eq. 3 is accurate for a range of temperature distributions, from global thermal equilibrium to local thermal equilibrium at a temperature expected in the absence of electron screening. It can be shown analytically that Eq. 3 has the correct long-time limit for when the plasma reaches global equilibrium, or when the expansion velocity greatly exceeds the thermal velocity. We do not believe our analysis is very sensitive to a particular assumption of $T_{i}(r)$.

In Fig. 2 using Eq. 3 we extract $T_{i, a v e}$ and $T_{e}$ from the data. The model addresses the dynamics after local equilibration, not the disorder-induced heating phase during the first $100 \mathrm{~ns}$. The fit $T_{e}$ exceed $2 E_{e} / 3 k_{b}$ for the data shown. As predicted by theory $[17,18,24,26]$, this effect becomes larger as the initial Coulomb coupling parameter for the electrons, $\Gamma_{e}=\kappa^{3} / 3=e^{2} /\left(4 \pi \varepsilon_{0} a k_{B} T_{e}\right)$, approaches unity. ( $\Gamma_{e}=0.5$ for the coldest electron temperature data in Fig. 2, assuming $T_{e}=2 E_{e} / 3 k_{B}$ and using the peak density.) Likely mechanisms for heating are recombination, continuum lowering, and disorder-induced electron heating. We plan to study this phenomenon in the future, but our main goal now is accurate determination of $T_{i, \text { ave }}$.

The inferred value of $T_{i, \text { ave }}$, which is the average ion temperature after the plasma reaches local thermal equilibrium, is shown in fig 3 . The uncertainty in $T_{i, \text { ave }}$ of about $0.2 \mathrm{~K}$, is determined by the statistical noise in the data and the uncertainty in $C$. We also show the theoretical value of $T_{i, a v e}$, found by averaging Eq. 2 over the density distribution. Uncertainty in this quantity results from uncertainty in the value of the peak density. Experimental and theoretical results agree well. It is not initially surprising that Eq. 2 accurately predicts the ion temperature for equilibrating ultracold neutral plasmas. The theory basically expresses energy conservation, as emphasized in [12]. However, for the data in Fig. 3 with the lowest fit $T_{e}$ and highest $n_{0 i}$, the peak value of $\kappa$ in the plasma is 0.7. This corresponds to three electrons per

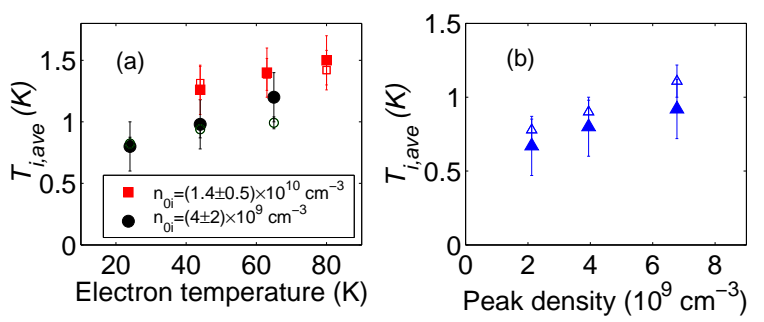

FIG. 3: The comparison between the data and the theoretical calculations. In (a), the density is fixed and the initial $T_{e}$ is varied. Closed symbols are experimental data and open symbols are theoretical calculations. In (b), the initial $T_{e}$ is fixed $(40 \mathrm{~K})$ while the density is varied. The $T_{e}$ for all theoretical calculations are based on the fit $T_{e}$
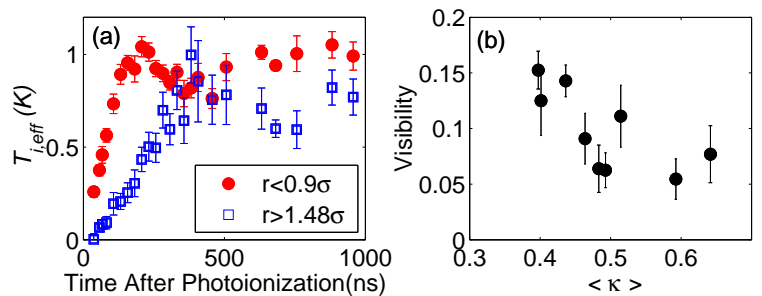

FIG. 4: Effective ion temperature obtained from different selected regions of the cloud for $n_{0 i}=(4 \pm 2) \times 10^{9} \mathrm{~cm}^{-3}$ and $T_{e}=38 \pm 6 \mathrm{~K}$. In (b), we plot the visibility of the oscillation for the central probed region versus the averaged $\kappa$ for this region for all our data taken at different conditions.

Debye sphere $\left(\kappa^{-3}=n_{e} 4 \pi \lambda_{D}^{3} / 3\right)$. One might not necessarily expect Eq. 2 to be accurate in this regime because it assumes a Yukawa potential for ion-ion interactions. The Yukawa potential is based on Debye screening and is normally derived for $\kappa^{-3} \gg 1$.

From the measured $T_{i, \text { ave }}, T_{e}$, and density distribution, we calculate the average Coulomb coupling constant for Debye-screend ions, $\Gamma_{i, a v g}^{*}=$ $\left\langle\exp (-\kappa(r)) e^{2} /\left(4 \pi \varepsilon_{0} a(r) k_{B} T_{i, a v e}\right)\right\rangle$. For all the data shown in Fig. $3, \Gamma_{i, a v g}^{*}$ is in the range of 1.7 to 2.5 . With lower $T_{e}$ and higher density, $\Gamma_{i, a v e}^{*}$ is slightly higher. The surprisingly small variation in $\Gamma_{i, a v g}^{*}$ suggests that disorder-induced heating is a natural feedback mechanism that leads to equilibration just barely in the strongly coupled regime.

Close inspection of Figs. 1 and 2 reveals that at the end of the disorder-induced heating phase, the ion temperature overshoots it equilibrium value before settling. This phenomenon is more evident in Fig. 4(a), where $T_{i, \text { ave }}$ is calculated for an inner and outer region of the plasma image $\left(\rho=\sqrt{x^{2}+y^{2}}<0.9 \sigma\right.$ and $\rho>1.48 \sigma$ respectively). Each selected annular region contains $1 / 3$ of the ions and probes a region with significantly less variation in density than in the entire plasma. The region with lower density has lower $T_{i, a v e}$, supporting our hypothesis of local thermal equilibrium, but the oscillation is the most striking observation.

Intuitively, one can explain this phenomenon as an oscillation of each ion in its local potential energy well. A simple calculation implies that the time for an ion to move an interparticle distance, when accelerated from rest by a force of $e^{2} /\left(4 \pi \varepsilon_{0} a^{2}\right)$, varies as $\sim \omega_{p i}^{-1}$, and the observed oscillation occurs at $2 \omega_{p i}$. As expected from the density dependence of $\omega_{p i}$, the oscillation period is longer for the outer region where the average density is lower. This explains why averaging over the entire cloud obscures the oscillation; the motion dephases because of the variation in $\omega_{p i}$. It is questionable whether the ion motion should be called an ion plasma oscillation or not, because there is probably no collective, or long range coherence to the motion.

Another way to think of the oscillation is relaxation of the two-particle distribution function in the plasma, 
which describes two-particle spatial and momentum correlations. Disorder-induced heating and oscillation of the kinetic and potential energy represent equilibration of spatial correlations. Normally, the time scale for equilibration of the two-particle distribution function is much shorter than the time scale for the one-particle distribution function to reach local thermal equilibrium. This is known as Bogoliubov's hypothesis [27]. For stronglycoupled plasmas, however, these time scales both become equal to $\omega_{p i}^{-1}[9]$.

Kinetic energy oscillations at $2 \omega_{p i}$ have been observed in molecular dynamics simulations of equilibrating strongly-coupled systems $[9,11,12]$. Calculations $[6,28]$ also show oscillations in the velocity autocorrelation function in equilibrium strongly-coupled one-component plasmas. To our knowledge, this is the first experimental observation of the phenomenon.

Numerical results[9] suggest that the damping time for the oscillations is approximately $\pi / \omega_{p i}$ for $\Gamma_{i} \geq 5$, and that lower $\Gamma_{i}$ leads to faster damping. Because our analysis still averages over the z-axis of the plasma, which introduces dephasing into the observed oscillations, it is difficult to comment on the damping of the oscillation.
We have performed the annular analysis for all the data and we found a correlation between the visibility of the oscillation and $\kappa$, as shown in Fig. 4(b). The visibility is defined as $\left(T_{i, \text { eff }}^{\text {peak }}-T_{i, \text { eff }}^{\text {dip }}\right) /\left(T_{i, \text { eff }}^{\text {peak }}+T_{i, \text { eff }}^{\text {dip }}\right)$, where $T_{i, \text { eff }}^{\text {peak }}$ and $T_{i, e f f}^{\text {dip }}$ are effective ion temperatures at the peak and dip of the oscillation.

In conclusion, we have studied the equilibration of ultracold neutral plasmas. The screening effect of electrons on the final ion temperature is observed. The experiment and theory agrees very well. Oscillation in the kinetic energy of ions is observed, which is a characteristic of strongly coupled plasmas. This demonstrated that the ultracold neutral plasma is a suitable system to study the equilibration of strongly coupled systems. Better measurement scheme will be developed and will provide a more quantitative comparison on this oscillation with the theory.

This research was supported by the Department of Energy Office of Fusion Energy Sciences, National Science Foundation, Office for Naval Research, Research Corporation, Alfred P. Sloan Foundation, and David and Lucille Packard Foundation.
[1] H. M. V. Horn, Science 252, 1384 (1991).

[2] T. B. Mitchell, J. J. Bollinger, X. P. Huang, W. M. Itano, and D. H. E. Dubin, Phys. Plasmas 6, 1751 (1999).

[3] J. H. Chu and L. I, Phys. Rev. Lett. 72, 4009 (1994).

[4] M. Nantel, G. Ma, S. Gu, C. Y. Cote, J. Itatani, and D. Umstadter, Phys. Rev. Lett. 80, 4442 (1998).

[5] E. Springate, N. Hay, J. W. G. Tisch, M. B. Mason, T. Ditmire, M. H. R. Hutchinson, and J. P. Marangos, Phys. Rev. A 61, 063201 (2000).

[6] J. P. Hansen, E. L. Pollock, and I. R. McDonald, Phys. Rev. Lett 32, 277 (1974).

[7] H. Gould and G. F. Mazenko, Phys. Rev. Lett. 35, 1455 (1975).

[8] M. Bonitz, D. Semkat, and D. Kremp, Phys. Rev. E 56, 1246 (1997).

[9] G. Zwicknagel, Contrib. Plasma Phys. 39, 155 (1999).

[10] K. Morawetz, M. Bonitz, V. G. Morozov, G. Röpke, and D. Kremp, Phys. Rev. E 63, 020102 (2001).

[11] I. V. Morozov and G. E. Norman, J. Phys. A: Mah. Gen. 36, 6005 (2003).

[12] T. Pohl, T. Pattard, and J. M. Rost, J. Phys. B: At. Mol. Opt. Phys. 37, 183 (2004).

[13] T. C. Killian, S. Kulin, S. D. Bergeson, L. A. Orozco, C. Orzel, and S. L. Rolston, Phys. Rev. Lett. 83, 4776 (1999).

[14] C. E. Simien, Y. C. Chen, P. Gupta, S. Laha, Y. N. Martinez, P. G. Mickelson, S. B. Nagel, , and T. C. Killian, Phys. Rev. Lett. 92, 143001 (2004).

[15] M. S. Murillo, Phys. Rev. Lett. 87, 115003 (2001).
[16] S. B. Nagel, C. E. Simien, S. Laha, P. Gupta, V. S. Ashoka, and T. C. Killian, Phys. Rev. A 67, 011401 (2003).

[17] S. G. Kuzmin and T. M. O'Neil, Phys. Plasmas 9, 3743 (2002).

[18] A. N. Tkachev and S. I. Yakovlenko, Quantum Electronics 30, 1077 (2000).

[19] S. Hamaguchi, R. T. Farouki, and D. H. E. Dubin, Phys. Rev. E 56, 4671 (1997).

[20] D. H. E. Dubin and T. M. O. Neil, Rev. Mod. Phys. 71, 87 (1999).

[21] T. Pohl, T. Pattard, and J. M. Rost, physics/0405125.

[22] T. C. Killian, Y. C. Chen, P. Gupta, S. Laha, Y. N. Martinez, P. G. Mickelson, S. B. Nagel , A. D. Saenz, and C. E. Simien, physics/0407138.

[23] S. Kulin, T. C. Killian, S. D. Bergeson, and S. L. Rolston, Phys. Rev. Lett. 85, 318 (2000).

[24] F. Robicheaux and J. D. Hanson, Phys. Plasmas 10, 2217 (2003).

[25] T. Pohl, T. Pattard, and J. M. Rost, Phys. Rev. Lett. 92, 155003 (2004).

[26] S. Mazevet, L. A. Collins, and J. D. Kress, Phys. Rev. Lett. 88, 55001 (2002).

[27] D. R. Nicholson, Introduction to Plasma Theory (Krieger Publishing Company Malabar, Florida, 1992).

[28] J. P. Hansen, I. R. McDonald, and E. L. Pollock, Phys. Rev. A 11, 1025 (1975). 